\title{
Observations on the vibration of axially tensioned elastomeric pipes conveying fluid
}

\author{
Y L Zhang ${ }^{1}$, D G Gorman ${ }^{1}, \mathbf{J}$ M Reese ${ }^{1 *}$ and $\mathbf{J}$ Horacek $^{2}$ \\ ${ }^{1}$ Department of Engineering, University of Aberdeen, Aberdeen, Scotland, UK \\ ${ }^{2}$ Institute of Thermomechanics of the Academy of Sciences of the Czech Republic, Prague, Czech Republic
}

\begin{abstract}
A study of the effect of axial tension on the vibration of a single-span elastomeric pipe clamped at both ends conveying fluid has been carried out both experimentally and theoretically. A new mathematical model using a penalty function technique and the method of kinematic correction and fictitious loads has been developed. The influence of flowing fluid and axial tension on natural frequencies and mode shapes of the system has been described using this model and compared with experimental observations. Linear and non-linear dynamic response of the harmonically excited pipe has also been investigated for varying flow velocities and initial axial tensions.
\end{abstract}

Keywords: pipe conveying fluid, vibration, finite element method

\section{NOTATION}

$A_{\mathrm{f}}, A_{\mathrm{p}} \quad$ cross-sectional area of the fluid and pipe respectively

$\mathbf{c}_{i}, \mathbf{C}_{i} \quad i$ th elemental and assembly fluid flowing damping matrices respectively

D symmetric stress-strain matrix

$\boldsymbol{e}, \boldsymbol{e}_{0} \quad$ strain and initial strain tensor fields of the pipe respectively

$E, E I, I \quad$ Young's modulus, flexural rigidity and area moment of inertia respectively global coordinate systems respectively

$f_{i}^{\text {ld }} \quad$ elemental fictitious load vector

$G \quad$ shear modulus

$h_{i} \quad$ shape functions for the pipe domain,

$i=1,2, \ldots, 10$

H matrix of the shape function for the fluid domain

$\boldsymbol{k}_{i}, \boldsymbol{K}_{i} \quad i$ th elemental stiffness matrices in the local and global coordinate systems respectively

$K \quad$ shear coefficient of the pipe material

$l, L \quad$ pipe elemental length and pipe length respectively

$m_{\mathrm{f}}, m_{\mathrm{p}} \quad$ fluid and pipe masses per unit length respectively
$D_{\mathrm{e}}, D_{\mathrm{i}} \quad$ external and internal diameters respectively

$\boldsymbol{f}_{i}, \boldsymbol{F}_{i} \quad i$ th elemental force vectors in the local and $\mathbf{m}_{i}, \mathbf{M}_{i}$

$\mathbf{m}_{\mathrm{f}}, \mathbf{m}_{\mathrm{p}}$

n

$\mathbf{N}$

$p$

$\overline{\boldsymbol{p}}_{\mathrm{f}}, \overline{\boldsymbol{p}}_{\mathrm{p}}$

$\boldsymbol{r}, \dot{\boldsymbol{r}}, \ddot{\boldsymbol{r}}$

$\boldsymbol{t}$

$T_{0}$

$\mathbf{T}_{i}$

$u, \varphi, w$

$\boldsymbol{u}_{\mathrm{p}}, \dot{\boldsymbol{u}}_{\mathrm{p}}, \ddot{\boldsymbol{u}}_{\mathrm{p}}$

$\boldsymbol{u}_{\mathrm{f}}, \boldsymbol{u}_{\mathrm{r}}$

$U, V$

$x$

$\overline{\boldsymbol{X}}_{\mathrm{f}}, \overline{\boldsymbol{X}}_{\mathrm{p}}$

$w_{\mathrm{am}}, y_{\mathrm{am}}$

$\Gamma_{\mathrm{f}}, \Gamma_{\mathrm{p}}$ ith elemental mass matrices in the local and global coordinate systems respectively fluid and pipe inertia force-acceleration matrices respectively

unit outward vector normal to the pipe surface

matrix of shape function for the pipe

domain

fluid pressure within the pipe

vectors of the prescribed fluid and pipe

boundary traction respectively

vector field of nodal displacements

unit vector tangential to the pipe surface

initial axial tension of the pipe

coordinate transformation matrix of the $i$ th element, $i=1,2, \ldots, m$

displacements in the $x, \varphi$ and $y$ directions in Cartesian coordinates respectively

vector fields of the pipe displacement

velocity and acceleration respectively

vector fields of the fluid absolute and relative displacements respectively

fluid scalar velocity and velocity vector field respectively

coordinate along the longitudinal direction of the pipe element

vectors of the fluid and pipe body forces respectively

peak displacement amplitude and seismic displacement amplitude respectively

revision for publication on 22 March 1999.

* Corresponding author: Department of Engineering, University of

Aberdeen, Fraser Noble Building, King's College, Aberdeen AB24 $3 U E, U K$.

\section{fluid and pipe boundary conditions} respectively, $\Gamma=\Gamma_{\mathrm{p}} \bigcup \Gamma_{\mathrm{f}}$ 


$\begin{array}{ll}\xi & =x / l \\ \rho_{\mathrm{f}}, \rho_{\mathrm{p}} & \begin{array}{l}\text { densities of fluid and pipe respectively } \\ \varphi_{i}, \varphi_{i+1}\end{array} \\ \begin{array}{l}\text { cross-sectional rotation of } i \text { th and }(i+1) \text { th } \\ \text { nodes of the } i \text { th element respectively }\end{array} \\ \begin{array}{l}\text { excitation frequency and natural frequency } \\ \text { respectively }\end{array} \\ \Omega_{\mathrm{f}}, \Omega_{\mathrm{p}} \quad \begin{array}{l}\text { fluid and pipe domains respectively }\end{array}\end{array}$

\section{Subscripts}

f fluid quantities

$n \quad n$th vibration mode

$\mathrm{p} \quad$ pipe quantities

\section{Superscript}

transpose of matrix

\section{INTRODUCTION}

The vibration of elastomeric pipes under axial tension conveying fluid is a topic of considerable interest in civil, mechanical and bioengineering. A review of the dynamics of pipes conveying fluid was conducted by Païdoussis and Li [1] and Blevins [2]. It has been shown theoretically, and confirmed experimentally, that the natural frequencies of axially tensioned tubes conveying fluid decrease when the flow velocity increases or the initial axial tension decreases [3]. However, there have been fewer investigations reported on the effect of axial tension and flow velocity on modal shapes and phase variation along the span. Naguleswarn and Williams [4] investigated experimentally the effect of flow velocity and axial tension on the fundamental natural frequency and its phase variation, and confirmed their analytical predictions using the two-term Galerkin method. Chen and Roseberg [5] theoretically accounted for the phenomena of phase propagation upstream due to the effect of the Coriolis force; however, no experimental confirmation was presented. Jendrzejczyk and Chen [6] stated briefly, without experimental verification, that the Coriolis force causes phase distortion but does not contribute to damping since the system is conservative. These researchers also observed that the modal damping ratio increased slightly with increasing flow velocity for an acrylic tube, clamped at both ends, conveying water. However, these investigations were confined to the linear regime and were used to predict natural frequencies up to 60 per cent of the critical flow velocity at which the system loses stability by bifurcation [3].

On the other hand, for many dynamic problems, such as blood vessels in fingers, above-ground pipelines subjected to wind gusts, and spanning subsea pipelines excited by cross-flow current, the effect of non-linearity is too important to ignore [7]. Thurman and Mote $[\mathbf{8 , 9 ]}$ conducted a non-linear study on the vibration of pipes conveying fluid; equations of motion in both the lateral and the longitudinal directions were developed and explicitly analysed via a hybrid method. Non-linear analyses of pipes conveying fluid have been performed by Rouselet and Herrmann [10], and Edelstein et al. [11], but these theoretical studies were confined to non-conservative systems and were performed to evaluate the applicable range of the linear theory and response in the post-critical flow velocity range.

The present paper describes the vibratory characteristics of a single-span liquid-filled or empty pipe under various initial axial tensions undergoing one-dimensional harmonic seismic excitation. Mode coupling, node shift and frequencies within both the linear and the non-linear response regimes due to non-linear geometrical effects are investigated.

In general, when the pipe vibrates laterally and longitudinally, the fluid flow within the pipe is affected; conversely, the fluid interacts with the walls of the pipe and alters the vibratory characteristics of the system. In this study the fluid was considered to be incompressible and inviscid with a constant and uniform velocity profile. The pipe had a constant circular cross-sectional area. Numerical simulations were performed for comparison with experimental data.

\section{MODEL FORMULATION}

Consider steady fluid flow within an elastomeric pipe clamped at both ends, as shown in Fig. 1. Let the coordinate $x$ be in the pipe centre-line at equilibrium and $y$ normal to it. The seismic displacement $y_{0}$ will be identical at any point within the whole system.

It is assumed that the pipe material is linear, homogeneous and isotropic and that the motion of the pipe is planar. The strain energy is combined with the kinetic energy for the pipe domain $\Omega_{\mathrm{p}}$ to form the Lagrangian function as follows [12]:

$$
\begin{aligned}
\Pi_{\text {pipe }}= & \frac{1}{2} \int_{\Omega_{\mathrm{p}}}\left(\boldsymbol{e}+\boldsymbol{e}_{0}\right)^{\mathrm{T}} \mathbf{D}\left(\boldsymbol{e}+\boldsymbol{e}_{0}\right) \mathrm{d} \Omega_{\mathrm{p}} \\
& -\int_{\Omega_{\mathrm{p}}} \boldsymbol{u}_{\mathrm{p}}^{\mathrm{T}} \overline{\boldsymbol{X}}_{\mathrm{p}} \mathrm{d} \Omega_{\mathrm{p}}-\int_{\Gamma_{\mathrm{p}}} \boldsymbol{u}_{\mathrm{p}}^{\mathrm{T}} \overline{\boldsymbol{p}}_{\mathrm{p}} \mathrm{d} \Gamma_{\mathrm{p}}
\end{aligned}
$$

The strain field, with the assumption that the deformed curvature can be approximated by the second derivative of the flexural displacement, may be expressed as [13]:

$$
\begin{aligned}
\boldsymbol{e} & =\boldsymbol{e}_{\mathrm{L}}+\boldsymbol{e}_{\mathrm{NL}} \\
& =\left\{\frac{\partial u}{\partial x}, \frac{\partial \varphi}{\partial x}, \varphi+\frac{\partial w}{\partial x}\right\}^{\mathrm{T}}\left\{\frac{1}{2}\left(\frac{\partial w}{\partial x}\right)^{2}, 0,0\right\}^{\mathrm{T}}
\end{aligned}
$$




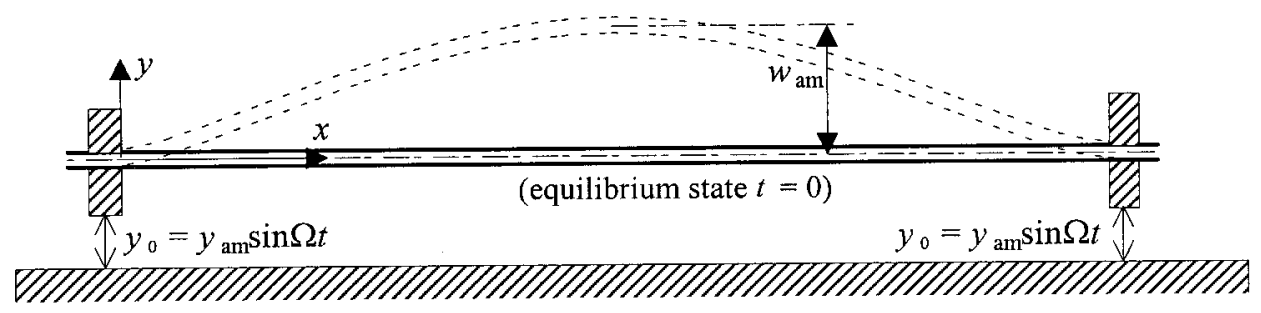

Fig. 1 Schematic diagram of the system

Applying the Hamilton principle [12] to equation (1), the following equation can be obtained:

$$
\begin{aligned}
\int_{\Omega_{\mathrm{p}}} & \mathbf{B}^{\mathrm{T}} \mathbf{D}\left(\mathbf{B} \boldsymbol{r}+\boldsymbol{e}_{0}\right) \mathrm{d} \Omega_{\mathrm{p}}+T_{0} \int_{\Omega_{\mathrm{p}}} \mathbf{N}_{\mathrm{w}}^{\prime \mathrm{T}} \mathbf{N}_{\mathrm{w}}^{\prime} \boldsymbol{r} \mathrm{d} \Omega_{\mathrm{p}} \\
& +\int_{\Omega_{\mathrm{p}}}\left(\mathbf{B}^{\mathrm{T}} \mathbf{D} \mathbf{B}_{\mathrm{NL}}+\mathbf{B}_{\mathrm{NL}}^{\mathrm{T}} \mathbf{D B}+\mathbf{B}_{\mathrm{NL}}^{\mathrm{T}} \mathbf{D} \mathbf{B}_{\mathrm{NL}}\right) \boldsymbol{r} \mathrm{d} \Omega_{\mathrm{p}} \\
& -\int_{\Omega_{\mathrm{p}}} \mathbf{N}^{\mathrm{T}} \overline{\boldsymbol{X}}_{\mathrm{p}} \mathrm{d} \Omega_{\mathrm{p}}-\int_{\Gamma_{\mathrm{p}}} \mathbf{N}^{\mathrm{T}} \overline{\boldsymbol{p}}_{\mathrm{p}} \mathrm{d} \Gamma_{\mathrm{p}}=\mathbf{0}
\end{aligned}
$$

where a prime denotes the first-order derivative with respect to $x$.

The equation describing the fluid motion in $\Omega_{\mathrm{f}}$ is

$$
\mathbf{m}_{\mathrm{f}} \frac{\mathrm{D} \boldsymbol{V}}{\mathrm{D} t}+\nabla p A_{\mathrm{f}}=\overline{\boldsymbol{X}}_{\mathrm{f}}
$$

together with the incompressibility condition

$$
\nabla \cdot \boldsymbol{V}=0
$$

where $\mathrm{D} / \mathrm{D} t$ is the material derivative of the fluid element. Galerkin's weighted residual method and Green's formula can be used to approximate the solution of equation (4):

$$
\begin{gathered}
\int_{\Omega_{\mathrm{f}}}\left(\mathbf{H m}_{\mathrm{f}} \frac{\mathrm{D} \boldsymbol{V}}{\mathrm{D} t}-p A_{\mathrm{f}} \mathbf{\nabla H}-\mathbf{H} \mathbf{m}_{\mathrm{f}}\right) \mathrm{d} \Omega_{\mathrm{f}} \\
-\int_{\Gamma_{\mathrm{f}}} \mathbf{H} \bar{p}_{\mathrm{f}} \mathrm{d} \Gamma_{\mathrm{f}}=0
\end{gathered}
$$

The Bubnov-Galerkin method is used to approximate the solution of a penalty function formulation of the continuity equation. The incompressible fluid is therefore approximated as slightly compressible:

$$
p=-\lambda(\boldsymbol{\nabla} \cdot \boldsymbol{V})=-\lambda \dot{u}^{\prime}
$$

where the superscript dot indicates a first-order partial derivative with respect to time and $\lambda$ is a very large constant penalty parameter; typically $\lambda \approx 10^{6}[\mathbf{1 4}]$. The vector field of the fluid acceleration can be expressed as

$$
\frac{\mathrm{D} \boldsymbol{V}}{\mathrm{D} t}=\frac{\partial^{2} \boldsymbol{u}_{\mathrm{p}}}{\partial t^{2}}+2 V \frac{\partial^{2} \boldsymbol{u}_{\mathrm{p}}}{\partial x \partial t}+V^{2} \frac{\partial^{2} \boldsymbol{u}_{\mathrm{p}}}{\partial x^{2}}
$$

Substituting equations (7) and (8) into equation (6) results in

$$
\begin{aligned}
\int_{\Omega_{\mathrm{f}}} & \mathbf{H m}_{\mathrm{f}}\left(\ddot{\boldsymbol{u}}_{\mathrm{p}}+2 V \dot{\boldsymbol{u}}_{\mathrm{p}}^{\prime}+V^{2} \boldsymbol{u}_{\mathrm{p}}^{\prime \prime}\right) \mathrm{d} \Omega_{\mathrm{f}} \\
& +\int_{\Omega_{\mathrm{f}}} \lambda A_{\mathrm{f}} \dot{\boldsymbol{u}}^{\prime} \nabla \mathbf{H} \mathrm{d} \Omega_{\mathrm{f}}-\int_{\Omega_{\mathrm{f}}} \mathbf{H} \overline{\boldsymbol{X}}_{\mathrm{f}} \mathrm{d} \Omega_{\mathrm{f}} \\
& -\int_{\Gamma_{\mathrm{f}}} \mathbf{H} \overline{\boldsymbol{p}}_{\mathrm{f}} \mathrm{d} \Gamma_{\mathrm{f}}=0
\end{aligned}
$$

In the interaction of the inviscid fluid with the pipe, only the normal compatibility is required while the slip condition is imposed, namely, only the normal component of the fluid displacement field is continuous and there exists a vector field of the fluid displacement relative to the pipe, $\boldsymbol{u}_{\mathrm{r}}$ :

$$
\begin{aligned}
& \left(\boldsymbol{u}_{\mathrm{p}}-\boldsymbol{u}_{\mathrm{f}}\right) \cdot \boldsymbol{n}=0 \\
& \left(\boldsymbol{u}_{\mathrm{p}}-\boldsymbol{u}_{\mathrm{f}}\right) \times \boldsymbol{n}+\boldsymbol{u}_{\mathrm{r}}=\mathbf{0}
\end{aligned}
$$

where $\boldsymbol{n}$ denotes the unit outward vector normal to the surface (from the pipe into the fluid).

In addition to the kinematic boundary conditions, the equilibrium of contact forces at the fluid-pipe interfaces should be imposed, namely the normal stress at the fluid-pipe interfaces is equal to the mean pressure in the fluid while no restriction is imposed on the tangential stresses:

$$
\begin{aligned}
& \boldsymbol{n} \cdot\left(\overline{\boldsymbol{p}}_{\mathrm{p}}+\overline{\boldsymbol{p}}_{\mathrm{f}}\right) \times \mathbf{n}=0 \\
& \boldsymbol{t} \cdot \overline{\boldsymbol{p}}_{\mathrm{p}} \times \boldsymbol{n}=0 \\
& \boldsymbol{t} \cdot \overline{\boldsymbol{p}}_{\mathrm{f}} \times \boldsymbol{n}=0
\end{aligned}
$$

where $t$ is the unit vector tangential to the surface.

The displacement field of the pipe may be expressed in terms of the shape function matrix $\mathbf{N}$, i.e., $\boldsymbol{u}_{\mathrm{p}}=\boldsymbol{u}_{0}+\mathbf{N} \boldsymbol{r}$ in which $\boldsymbol{r}$ and $\boldsymbol{u}_{0}$ represent the vectors of nodal displacements and the prescribed function of kinematic boundary conditions respectively. The terms in equations (2) and (5) containing contact forces at the fluidpipe interfaces can be eliminated when the test functions 
for the fluid and pipe are identical, i.e. $\mathbf{N}^{\mathrm{T}}=\mathbf{H}$. Using ordinary isoparametric finite elements for $\boldsymbol{u}_{\mathrm{p}}$ and $\boldsymbol{u}_{\mathrm{f}}$, adding equation (3) to equation (9), and applying boundary conditions and Green's theorem, the elemental equation governing the motion of the fluid-pipe system in the local coordinate system is

$$
\mathbf{m}_{i} \ddot{\boldsymbol{r}}+\mathbf{c}_{i} \dot{\boldsymbol{r}}+\mathbf{k}_{i} \boldsymbol{r}=\boldsymbol{f}_{i}+\boldsymbol{f}_{i}^{\mathrm{ld}}
$$

where $\mathbf{m}_{i}, \mathbf{c}_{i}$ and $\mathbf{k}_{i}$ are the elemental mass, damping and stiffness matrices associated with the inertia, Coriolis and centrifugal forces respectively and represent the effect of the liquid on the pipe and vice versa; $\boldsymbol{r}, \dot{\boldsymbol{r}}$ and $\ddot{\boldsymbol{r}}$ represent the vector fields of nodal displacements, velocities and accelerations respectively of the pipe; $\boldsymbol{f}_{i}$ and $\boldsymbol{f}_{i}^{\mathrm{ld}}$ are the vectors of nodal forces and fictitious loads associated with kinematic correction respectively. These elemental matrices and vectors are listed in the Appendix and the other notation is detailed at the beginning of the paper. The moving pipe and flowing fluid are fully coupled through the compatibility of displacement and the equilibrium of contact forces. In this analysis, the Eulerian approach and the concept of fictitious loads based on the in-plane kinematic corrections $[15,16]$ due to large deformation are applied to equation (12). The following set of differential equations in the global coordinate system is obtained:

$$
\mathbf{M}_{i} \ddot{\boldsymbol{r}}+\mathbf{C}_{i} \dot{\boldsymbol{r}}+\mathbf{K}_{i} \boldsymbol{r}=\boldsymbol{F}_{i}
$$

where

$\mathbf{M}_{i}=\mathbf{T}_{i}^{\mathrm{T}} \mathbf{m}_{i} \mathbf{T}_{i}$, the structural mass matrix $\mathbf{C}_{i}=\mathbf{T}_{i}^{\mathrm{T}} \mathbf{c}_{i} \mathbf{T}_{i}$, the structural stiffness matrix

$$
\begin{aligned}
& \mathbf{K}_{i}=\mathbf{T}_{i}^{\mathrm{T}} \mathbf{k}_{i} \mathbf{T}_{i}, \text { the structural stiffness matrix } \\
& \boldsymbol{F}_{i}=\mathbf{T}_{i}^{\mathrm{T}}\left(\boldsymbol{f}_{i}+\boldsymbol{f}_{i}^{\mathrm{ld}}\right), \text { the forcing vector }
\end{aligned}
$$

Note that, when assembling elemental matrices and vectors, the nodal forces are common to adjacent elements and the added values are zero except at the locations of concentrated or equivalent forces, or moments. As the effects of shear deformation and rotary inertia are incorporated in equation (13), the linearized version of this equation may be adopted to conduct the analysis of the vibration of a Timoshenko beam. The transient response of this system can be straightforwardly solved by using a Newmark [17] method and eigenvalues can be extracted using the inverse simultaneous iteration method after linearizing the assembled equations.

\section{EXPERIMENTAL INVESTIGATION}

In order to make a quantitative comparison, tests were performed on a single-span elastomeric pipe conveying fluid (water) which was supported at both ends on a vibrating platform. The pipe was mounted vertically as shown in Fig. 2 to enable vibration measurements to be performed using a laser velocity transducer, and to alleviate any lateral gravitational effects. Vibratory response at several positions along the pipe was measured by re-aligning the laser velocity transducer in order to concentrate its beam at a specific point on the pipe. The excitation and response signals, from the accelerometer and laser velocity transducer (integrated to displacement) respectively, were then processed by a real-time fast Fourier transform analyser.

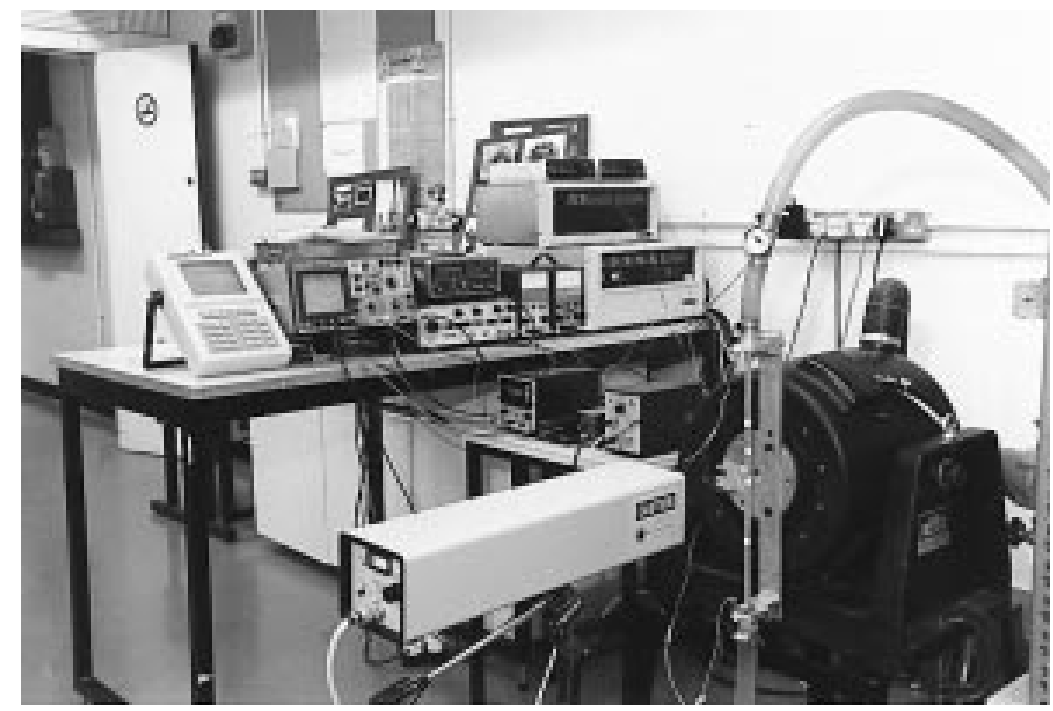

Fig. 2 Experimental apparatus 
The frequency of excitation was adjusted until the phase shift between these two signals was $\pm 90^{\circ}$; this indicated the resonant frequency in the linear regime. This process was completed for various initial axial tensions, flow velocities and several amplitudes of seismic excitation. It was observed that at the lower amplitudes of seismic excitation the pipe exhibited linear response behaviour, as expected. As the amplitude of seismic excitation is increased at the same frequency, non-linear behaviour of the pipe appears.

With the gradual increase in the amplitude of seismic excitation, it was observed that there existed a critical amplitude above which the vibration of the pipe conveying fluid exhibited non-linear response characteristics with two stable regions in the frequency response graph, as shown in Fig. 3. The lower stable region is obtained if the excitation frequency is decreased gradually; conversely the upper stable region is obtained if the frequency of excitation increases. The dynamic response curve is skewed at the upper resonant frequency of $32.16 \mathrm{~Hz}$.

\section{RESULTS AND DISCUSSION}

Consider the case where the system is harmonically excited by seismic excitation of the end supports: $y_{0}(t)=y_{\text {am }} \sin (\Omega t)$, where $\Omega$ is the circular frequency of the harmonic motion and $y_{\mathrm{am}}$ is the amplitude. The properties of components used in the experiment and numerical simulation are listed in Table 1 . For the numerical simulation performed, the system was discretized into 16 elements.

For differing axial tensions, flow velocities and vibration modes, there are different critical seismic
Table 1 Properties of components used in the experiment and numerical simulation

\begin{tabular}{ll}
\hline Length $L$ & $3.62 \times 10^{-1} \mathrm{~m}$ \\
Internal diameter $D_{\mathrm{i}}$ & $6.0 \times 10^{-3} \mathrm{~m}$ \\
External diameter $D_{\mathrm{e}}$ & $9.7 \times 10^{-3} \mathrm{~m}$ \\
Young's modulus $E$ & $2.0924 \times 10^{6} \mathrm{~N} / \mathrm{m}^{2}$ \\
Poisson's ratio $\nu$ & 0.5 \\
Pipe density $\rho_{\mathrm{p}}$ & $1128.56 \mathrm{~kg} / \mathrm{m}^{3}$ \\
Fluid density $\rho_{\mathrm{f}}$ & $1000.0 \mathrm{~kg} / \mathrm{m}^{3}$ \\
\hline
\end{tabular}

excitation amplitudes above which a non-linear response prevails and vice versa. The pipe conveying fluid is modelled for clamped-clamped boundary conditions. The natural frequencies were predicted and measured for the pipe with various flow velocities and initial axial tensions within the linear regime. Figure 4 shows the effect of axial tension on the first- and third-mode natural frequencies in the linear regime. The comparison of theoretical and experimental results for the pipe in the absence of or containing quiescent fluid is shown. Note that, as expected, the natural frequencies increase with increasing axial tension.

Figure 5 shows the effect of flow velocity on the first-, third- and fifth-mode natural frequencies. For higher modes, the experimental natural frequencies would appear to be higher than the theoretical values. The maximum difference in natural frequencies between prediction and experiment is 9 per cent; such discrepancies may be due to experimental errors and the axioms of the mathematical model, namely (a) non-ideal clamping conditions, (b) the fact that fluid flow was considered as having a uniform flow profile and (c) the fact that the properties of the rubber were approximated by assuming a linear elastic response. It is seen from

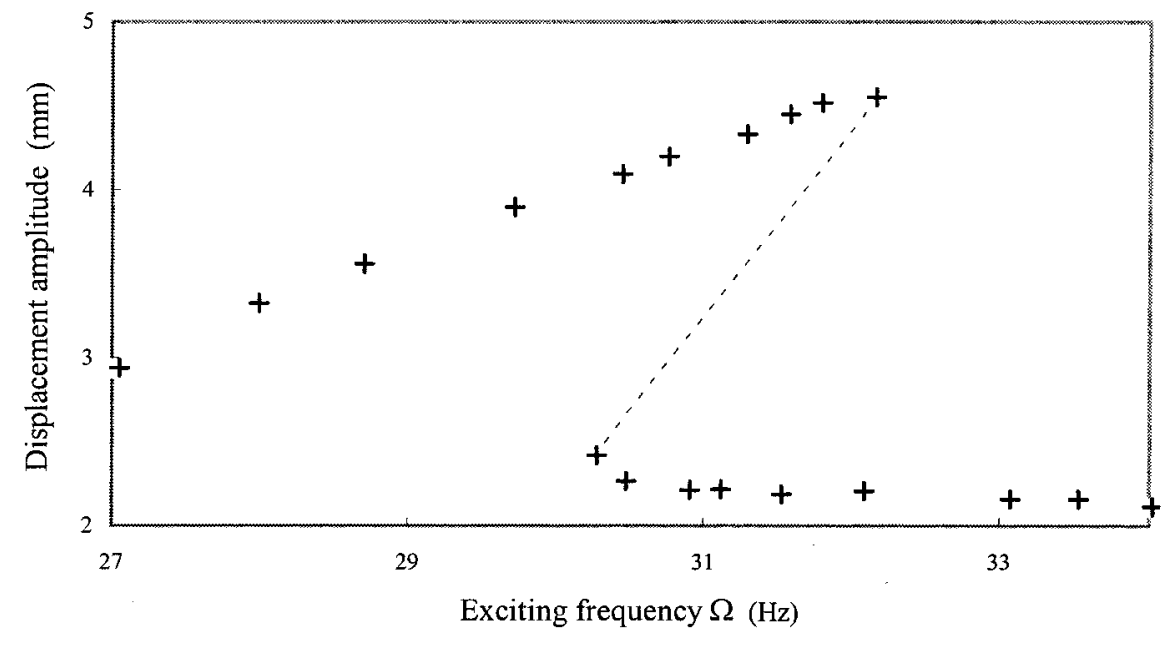

Fig. 3 Experimental dynamic response at $x / L=0.1$ within the non-linear response regime for the pipe containing quiescent fluid at $T_{0}=7.63 \mathrm{~N}$ and $y_{\mathrm{am}}=0.00032 \mathrm{~m}$ 

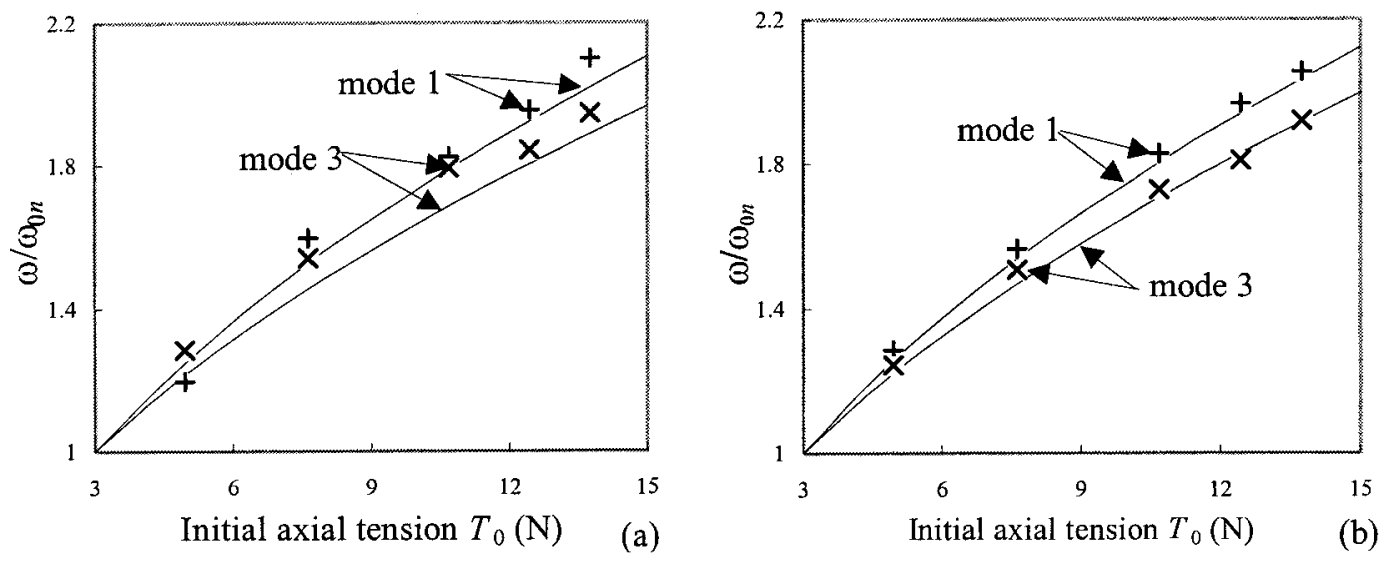

Fig. 4 Comparison of theoretical natural frequencies with experiment for various axial tensions (a) in the absence of fluid $\left(\omega_{01}=11.67 \mathrm{~Hz}\right.$ and $\left.\omega_{03}=26.25 \mathrm{~Hz}\right)$; and (b) with quiescent fluid $\left(\omega_{01}=9.30 \mathrm{~Hz}\right.$ and $\left.\omega_{03}=30.11 \mathrm{~Hz}\right):-$, theoretical results;,$+ \times$, experiment

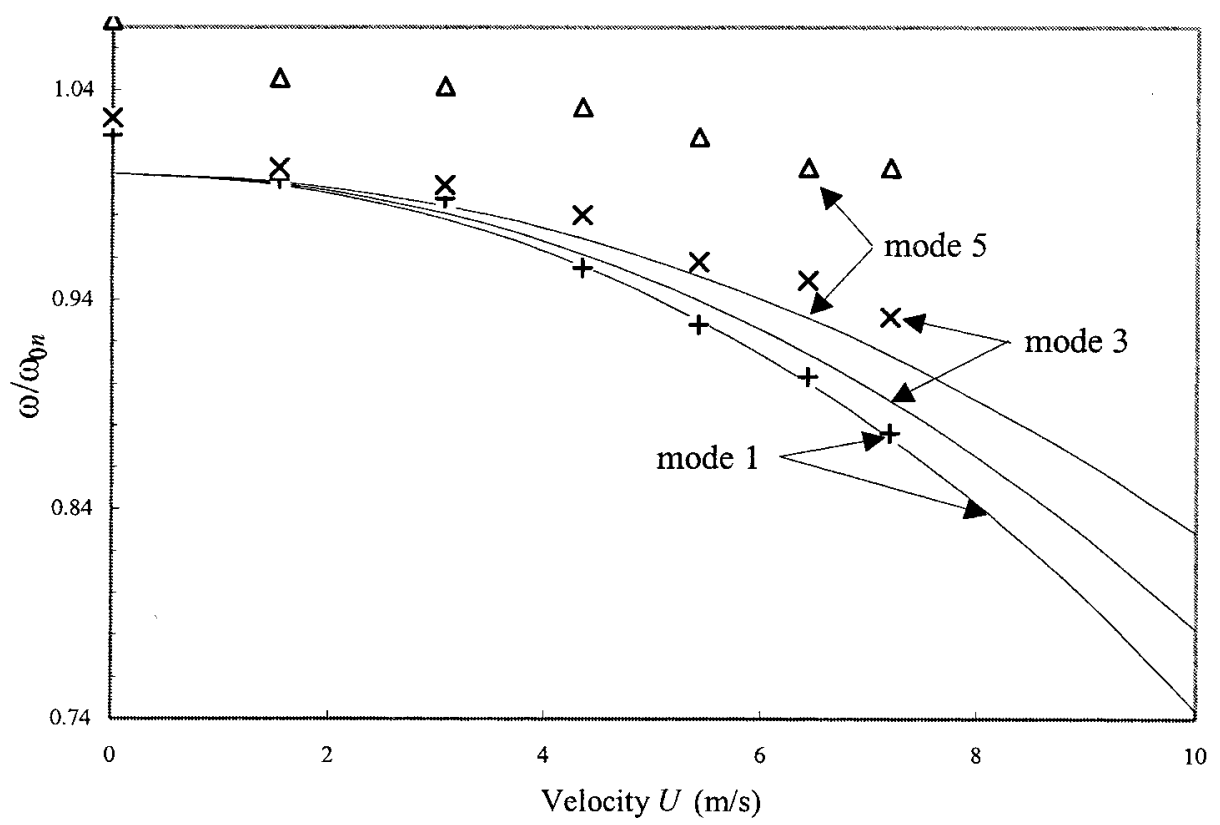

Fig. 5 Comparison of theoretical natural frequencies with experiment at $T_{0}=7.63 \mathrm{~N}$ for internal flow velocities $\left(\omega_{01}=14.30 \mathrm{~Hz}, \omega_{03}=44.23 \mathrm{~Hz}\right.$ and $\left.\omega_{05}=77.49 \mathrm{~Hz}\right)$ : - theoretical results;,$+ \times, \triangle$, experiment

Figs 4 and 5 that the frequencies obtained by this model are in good agreement with the experiment.

The experimental dynamic response for several flow velocities within the linear regime is compared with theoretical predictions in Fig. 6. An increase in the flow velocity leads to a decrease in the vibration amplitude. It is also seen that the lateral displacements obtained by this model are in good agreement with experiment.

Figure 7 a shows the calculated response over 1 cycle of transverse vibration of the pipe containing static fluid for the first mode within the non-linear regime. This vibration exhibits a classical mode. When the flow velocity increases, the fluid exerts a great effect on modal shape. The phase variation along the pipe span is shown in Fig. $7 \mathrm{~b}$ and the phase is seen to propagate upstream due to the effect of the Coriolis force. The theoretical predictions are confirmed by experiment.

Almost all points of the pipe vibrate relatively with different phases. This will be further illustrated in the complex plane below. Such shapes display travelling 


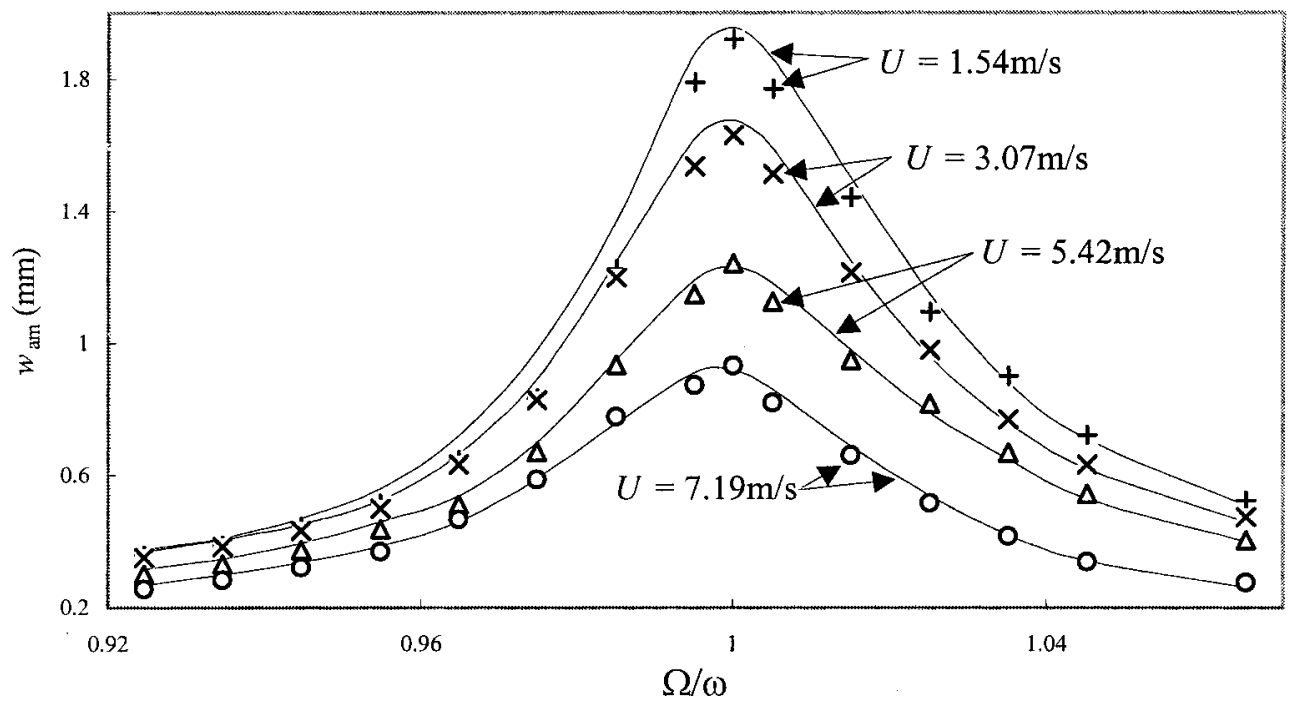

Fig. 6 Linear dynamic response of the pipe conveying fluid for the third mode at $T_{0}=7.63 \mathrm{~N}$ and $y_{\mathrm{am}}=0.00087 \mathrm{~m}:-$, theoretical results; $+, \times, \triangle, \circ$, experiment
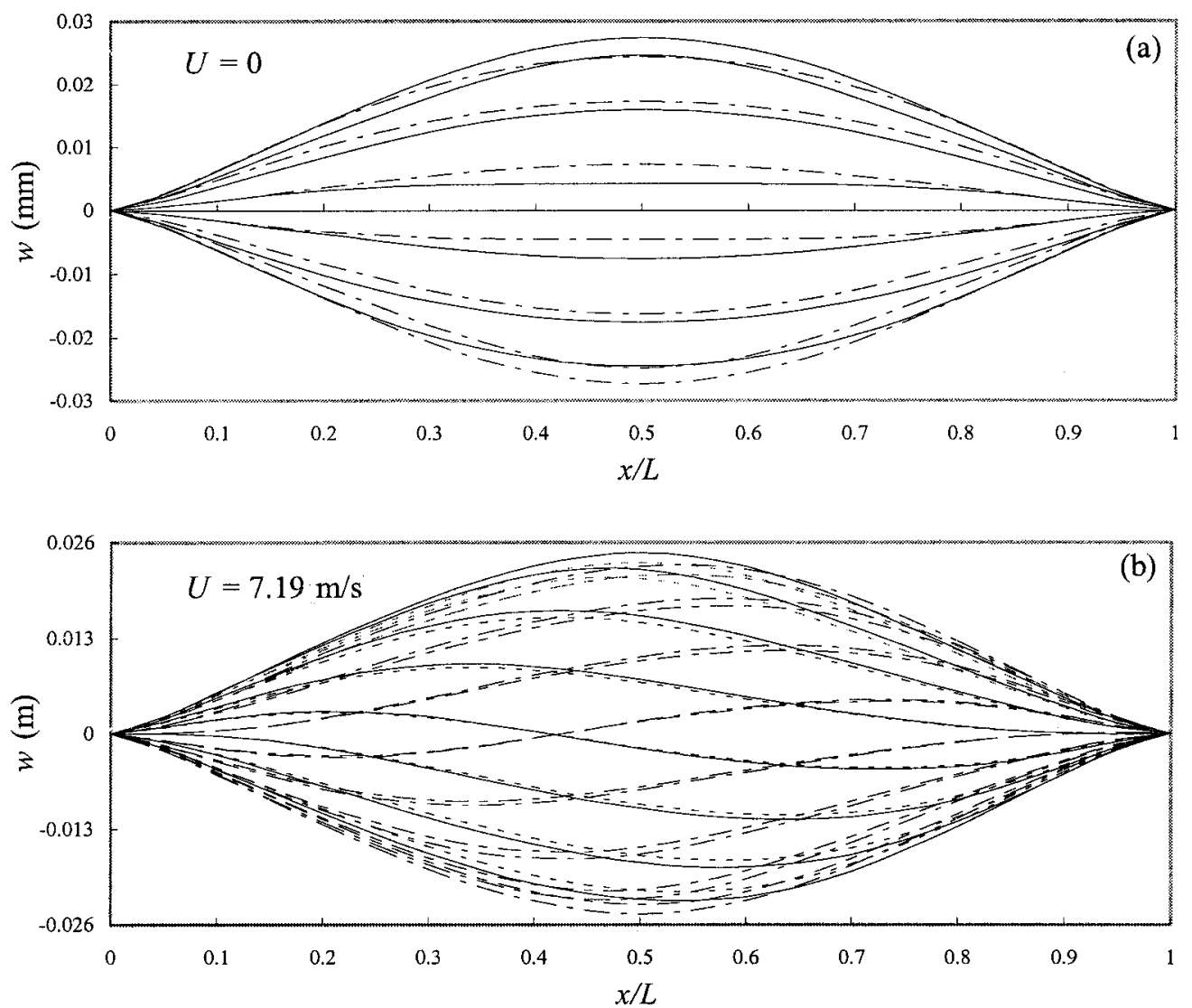

Fig. 7 Cycle of the lateral vibration of the pipe conveying fluid for the first mode at $T_{0}=7.63 \mathrm{~N}$ and $y_{\mathrm{am}}=0.00265 \mathrm{~m}$ for (a) $U=0 \mathrm{~m} / \mathrm{s}$ and (b) $U=7.19 \mathrm{~m} / \mathrm{s}$ ( $x$ denote the distance between the upstream end of the pipe and the node): - , theoretical results, downward motion; - - -, theoretical results, upward motion; - - - -, experiment, downward motion; --, experiment, upward motion 

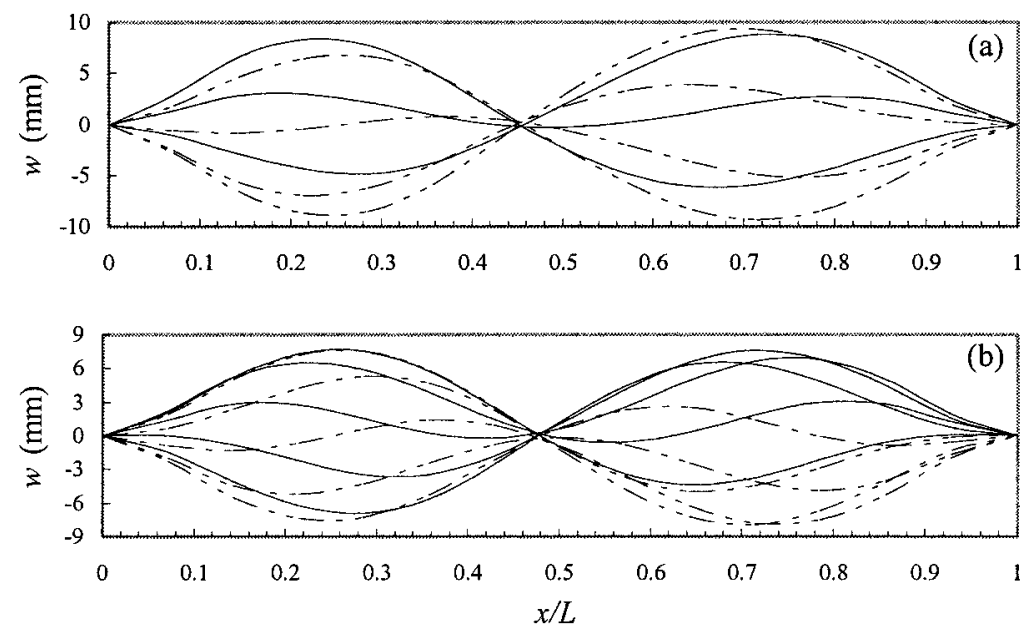

Fig. 8 Cycle of the lateral vibration of the pipe conveying fluid for the second mode at $T_{0}=7.63 \mathrm{~N}$ and $y_{\mathrm{am}}=0.00032 \mathrm{~m}$, for (a) $U=5 \mathrm{~m} / \mathrm{s}$ and (b) $U=10 \mathrm{~m} / \mathrm{s}$ : - , downward motion; - - -, upward motion

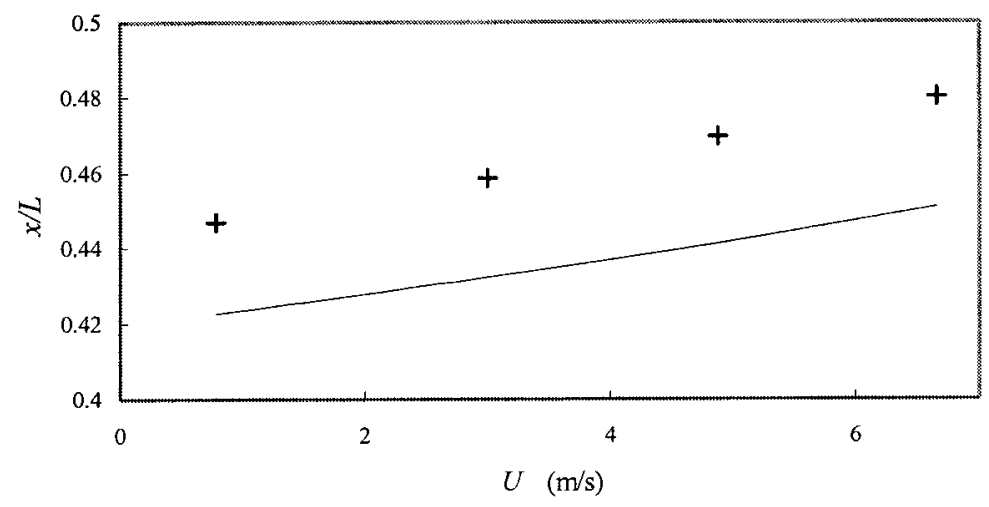

Fig. 9 Nodal position dependence on internal flow velocity for the second mode in the non-linear regime at $T_{0}=7.63 \mathrm{~N}$ and $y_{\mathrm{am}}=0.00032 \mathrm{~m}:+$, experiment; - , theoretical results

wave forms; the speed of these waves is directly related to the flow velocity. Because of the Coriolis term, the damping matrix becomes skew symmetric, and the nodes of the modes move also with the change in flow velocity.

Figure $7 \mathrm{~b}$ also shows that the pipe conveying fluid has a dynamic response behaviour characterized by firstand second-mode coupling, albeit dominated by the first mode.

For the pipe supported at both ends, the second mode has only one vibration node. Figure 8 shows that the dynamic response behaviour of the pipe containing flowing fluid, although dominated by the second mode, has strong components from the second and third modes. It is also clearly shown in Figs $8 \mathrm{a}$ and $\mathrm{b}$ that the effect of increasing flow velocity is to shift the node downstream. When the fluid within the pipe is stationary, the second- and even-order modes disappear due to the nature of the symmetric seismic excitation described above. This behaviour was verified experi- mentally. Figure 9 shows a comparison of theoretical results of the node motion with experimental values for the second vibratory mode. The experimental values are only a little higher than those predicted.

Figure 10 shows the experimental results of nodal shift with respect to the flow velocity within the linear and non-linear response regimes. This figure also clearly shows that a vibration node moves forwards further with increasing flow rate within the non-linear response regime than in the linear regime.

As the flow velocity increases, the interaction between the pipe and fluid becomes more pronounced. Accordingly, in view of the strong influence of Coriolis effects, the eigenvectors are characterized not only by the amplitudes but also by the phase. The calculated eigenvectors for the first mode on the complex plane are shown in Fig. 11.

The angle between two tangent lines of any two points on the eigenvector curve represents the phase difference between these two points. Figure 11 also shows the 

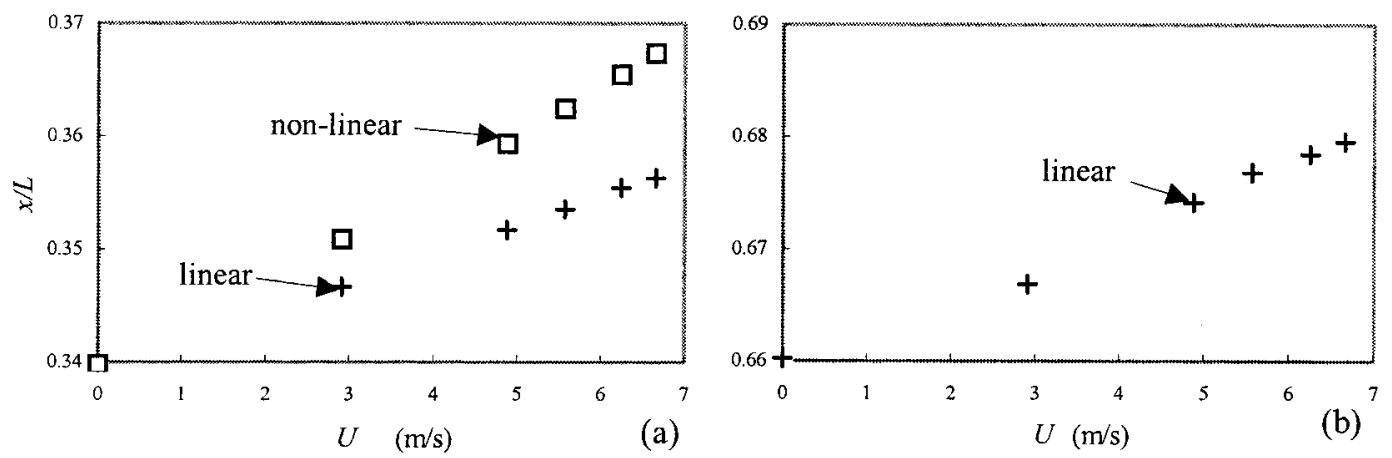

Fig. 10 Nodal position dependence on the internal flow velocity for the third mode within the linear $\left(y_{\mathrm{am}}=0.00022 \mathrm{~m}\right)$ and non-linear regime $\left(y_{\mathrm{am}}=0.00027 \mathrm{~m}\right)$ at $T_{0}=7.63 \mathrm{~N}$ for (a) the upstream node and (b) the downstream node

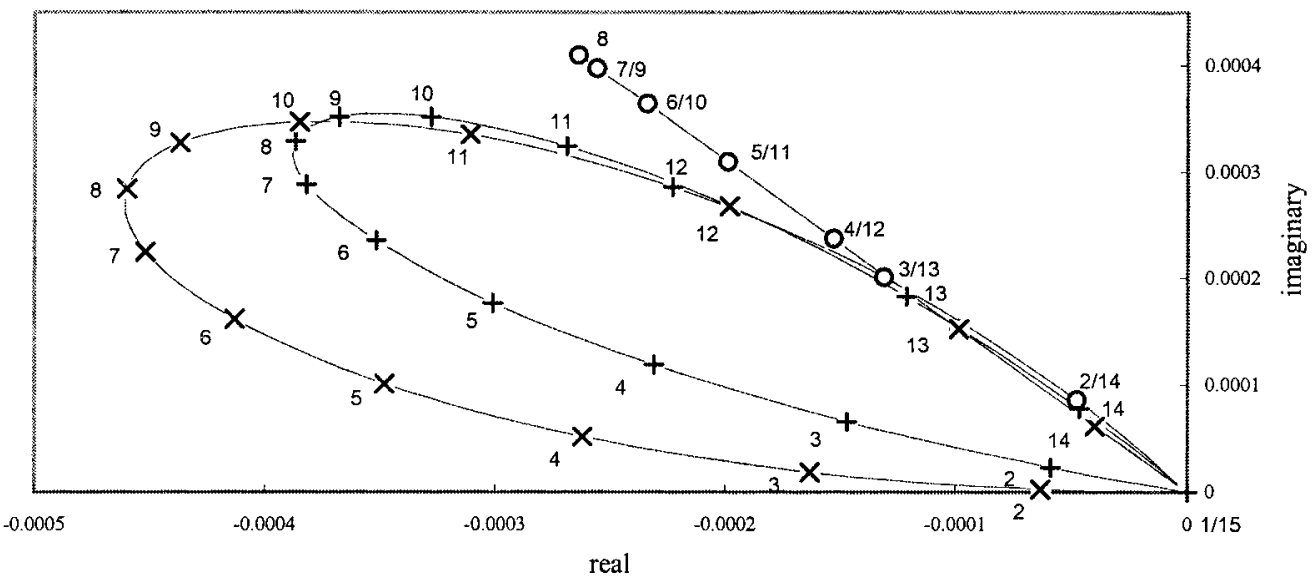

Fig. 11 First lateral mode shape in the complex plane at $T_{0}=7.63 \mathrm{~N}$, where the total number of nodes is 15 : ${ }_{-\circ}, U=0.0 \mathrm{~m} / \mathrm{s} ;-+-, U=7.19 \mathrm{~m} / \mathrm{s} ;-\times-, U=10 \mathrm{~m} / \mathrm{s}$

upper branch of the complex eigenvector 'ellipse', gradually moving towards a straight line as the flow velocity decreases. For $U=0.0$, the two branches coincide in a straight line, which implies that the phase shift for any point along the pipe is zero. With increase in the flow velocity, all points of the pipe vibrate with increasing phase shift. Both modes in Fig. 11 are coupled to other modes for all cases where $U \neq 0.0$.

Figure 12 highlights the effect of axial tension on the first- and second-mode shapes of the pipe. As the axial tension increases (the pipe becomes stiffer), the mode shape flattens and the phase shift tends to be smaller. The modes of lateral vibrations dominated by the coupled first and second modes were weakly coupled to other modes as axial tension increased.

\section{CONCLUSION}

A finite element model has been developed to solve dynamic problems of pipes conveying fluid, using a penalty function technique and fictitious loads based on the kinematic energy correction. This model can be conveniently applied to both linear and non-linear vibration analysis. The dynamic behaviour of an elastomeric pipe containing flowing or quiescent fluid (or in the absence of fluid), clamped at both ends, and under an axial tension was examined. The matrix equations of motion governing both the transverse and the longitudinal vibrations were solved by using the Newmark method. Mode coupling for the pipe conveying fluid was examined numerically and experimentally. In the case of the pipe containing flowing fluid, it was found that all excited modes were strongly coupled to other modes.

Experimental investigations were performed for the first three odd modes of vibration. Linear-regime natural frequencies were observed to decrease as the axial tension decreases or the flow velocity increases. It was also observed, theoretically and experimentally, that the vibrating nodes shifted downstream as the flow velocity increases. A further finding was that the third-mode vibration nodes move further forwards within the non- 

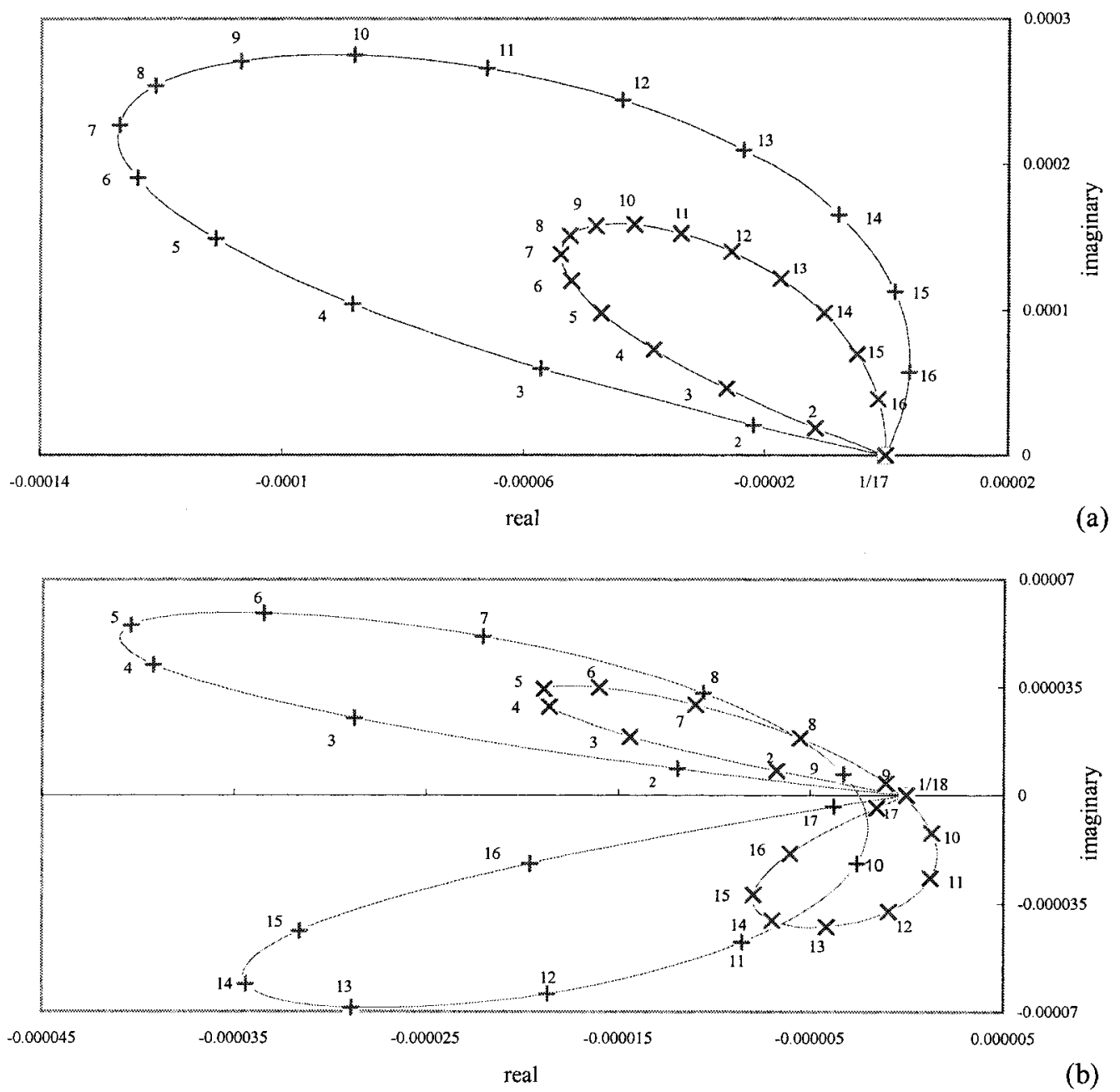

Fig. 12 Effect of the axial tension on the lateral mode shapes for (a) the first mode and (b) the second mode in the complex plane at $U=5.0 \mathrm{~m} / \mathrm{s}$, where the total number of nodes is 17 or 18 ; - + $T_{0}=4.95 \mathrm{~N} ;-\times-, T_{0}=14.85 \mathrm{~N}$

linear response regime than in the linear regime, and also with increasing flow velocity. The experimental results showed good agreement with the theory presented.

\section{ACKNOWLEDGEMENTS}

The research was supported by The Royal Society (London), the University of Aberdeen and The Grant Agency of the Czech Republic, Project 101/97/0826.

\section{REFERENCES}

1 Païdoussis, M. P. and Li, G. X. Pipes conveying fluid: a model dynamical problem. J. Fluids Structs, 1993, 7, 137204.

2 Blevins, R. D. Flow-Induced Vibration, 2nd edition, 1990 (Van Nostrand Reinhold, New York).

3 Lui, H. S. and Mote Jr, C. D. Dynamic response of pipes transporting fluids. Trans. ASME, J. Engng Industry, 1974, 96, 591-596.

4 Naguleswarn, S. and Williams, C. J. H. Lateral vibrations of a pipe conveying fluid. J. Mech. Engng Sci., 1968, 10, 228-238.

5 Chen, S. S. and Rosenberg, G. S. Vibrations and stability of a tube conveying fluid. Report ANL-7762, Argonne National Laboratory, 1971.

6 Jendrzejczyk, J. A. and Chen, S. S. Experiments on tubes conveying fluid. Thin-walled Structs, 1985, 3, 109-134.

7 Holmes, P. J. Pipes supported at both ends cannot flutter. J. Appl. Mechanics, 1978, 45, 619-622.

8 Thurman, A. L. and Mote Jr, C. D. Free, periodic nonlinear oscillation of an axially moving strip. J. Appl. Mechanics, 1969, 36, 83-91.

9 Thurman, A. L. and Mote Jr, C. D. Nonlinear oscillation of a cylinder containing flowing fluid. Trans. ASME, J. Engng Industry, 1969, 91, 1147-1155.

10 Rousselet, J. and Herrmann, G. Dynamic behavior of continuous cantilevered pipes conveying fluid near critical velocities. J. Appl. Mechanics, 1981, 48, 943-947.

11 Edelstein, W. S., Chen, S. S. and Jendrzejczyk, J. A. A 
finite element computation of the flow-induced oscillations in a cantilevered tube. J. Sound Vibr., 1986, 107(1), 121129.

12 Bittnar, Z. and Sejnoha, J. Numerical Methods in Structural Mechanics, 1996, pp.45-52 (American Society of Civil Engineers, New York).

13 Zienkiewicz, O. C. The Finite Element Method, 3rd edition, 1982, pp. 449-556 (McGraw-Hill, New York).

14 Noronha, W. P. and Berman, J. A finite element approximation of steady flow through a rotating nonaligned straight tube. Int. J. Numer. Meth. Fluids, 1986, 6, 733-747.

15 Zhang, Y. L., Gorman, D. G. and Reese, J. M. Analysis of the vibration of pipes conveying fluid. Proc. Instn Mech. Engrs, Part C, Journal of Mechanical Engineering Science, 1999, 213(C8), 849-860.

16 Kohnke, P. C. Large deflection analysis of frame structures by fictitious forces. Int. J. Numer. Meth. Engng, 1978, 12, 1279-1294.

17 Newmark, N. M. A method of computation for structural dynamics. J. Engng Mechanics Div., ASCE, 1959, 85, 6794.

\section{APPENDIX}

\section{Elemental matrices and vectors}

$$
\begin{aligned}
\mathbf{m}_{i}= & l \int_{0}^{l} \mathbf{N}^{\mathrm{T}}\left(\mathbf{m}_{\mathrm{p}}+\mathbf{m}_{\mathrm{f}}\right) \mathbf{N} \mathrm{d} \xi \\
\mathbf{c}_{i}= & U l \int_{0}^{l} \mathbf{N}^{\mathrm{T}} \mathbf{m}_{\mathrm{f}} \mathbf{N}^{\prime} \mathrm{d} \xi-U l \int_{0}^{l} \mathbf{N}^{\prime \mathrm{T}} \mathbf{m}_{\mathrm{f}} \mathbf{N} \mathrm{d} \xi \\
& +\lambda A_{\mathrm{f}} l \int_{0}^{L} \mathbf{N}_{u}^{\mathrm{T}} \mathbf{N}_{u}^{\prime} \mathrm{d} \xi \\
\mathbf{k}_{i}= & l \int_{0}^{l} \mathbf{B}^{\mathrm{T}} \mathbf{D} \mathbf{B} \mathrm{d} \xi-U^{2} l \int_{0}^{l} \mathbf{N}^{\prime \mathrm{T}} \mathbf{m}_{\mathrm{f}} \mathbf{N}^{\prime} \mathrm{d} \xi \\
& +T_{0} l \int_{0}^{l} \mathbf{N}_{w}^{\prime \mathrm{T}} \mathbf{N}_{w}^{\prime} \mathrm{d} \xi \\
& -\left.\left(U \mathbf{N}^{\mathrm{T}} \mathbf{m}_{\mathrm{f}} \mathbf{N}_{\boldsymbol{r}}+U^{2} \mathbf{N}^{\mathrm{T}} \mathbf{m}_{\mathrm{f}} \mathbf{N}^{\prime} \boldsymbol{r}\right)\right|_{0} ^{l} \\
\boldsymbol{f}_{i}= & -l \int_{0}^{l} \mathbf{B}^{\mathrm{T}} \mathbf{D} \boldsymbol{e}_{0} \mathrm{~d} \xi+l \int_{0}^{l} \mathbf{N}^{\mathrm{T}}\left(\overline{\boldsymbol{X}}_{\mathrm{p}}+\overline{\boldsymbol{X}}_{\mathrm{f}}\right) \mathrm{d} \xi \\
& +l \int_{0}^{l} \mathbf{N}^{\mathrm{T}}\left(\overline{\boldsymbol{p}}_{\mathrm{p}}+\overline{\boldsymbol{p}}_{\mathrm{f}}\right) \mathrm{d} \xi \\
& -l \int_{0}^{l} \mathbf{N}^{\mathrm{T}}\left(\mathbf{m}_{\mathrm{p}}+\mathbf{m}_{\mathrm{f}}\right) \ddot{\boldsymbol{u}}_{0} \mathrm{~d} \xi \\
& \\
& \\
& \\
&
\end{aligned}
$$

$$
\begin{aligned}
\boldsymbol{f}_{i}^{\mathrm{ld}} & =\mathbf{T}_{i}^{T} \mathbf{k}_{i}^{0} \boldsymbol{u}^{\mathrm{ld}} \\
& =\mathbf{T}_{i}^{\mathrm{T}}\left\{-a_{1}, 2 a_{2}, a_{2} l, a_{3},-2 a_{4}, a_{4} l\right\}^{\mathrm{T}} \\
\boldsymbol{r} & =\left\{u_{1}, w_{1}, \varphi_{1}, u_{2}, w_{2}, \varphi_{2}\right\}^{\mathrm{T}}
\end{aligned}
$$

where

$$
\begin{aligned}
\mathbf{k}_{i}^{0}= & l \int_{0}^{l} \mathbf{B}^{\mathrm{T}} \mathbf{D B} \mathrm{d} \xi \\
\boldsymbol{u}^{\mathrm{ld}=} & \left\{0,0, \varphi_{j}-\sin \varphi_{j},\right. \\
& \left.l\left(1-\cos \varphi_{j+1}\right), 0, \varphi_{j+1}-\sin \varphi_{j+1}\right\}^{\mathrm{T}} \\
a_{1}= & A_{\mathrm{p}} E\left(1-\cos \varphi_{j}\right) \\
a_{2}= & \frac{6 E I\left(\varphi_{j}-\sin \varphi_{j}\right)}{l^{2}+12 E I / K G A_{\mathrm{p}}}
\end{aligned}
$$$$
a_{3}=A_{\mathrm{p}} E\left(1-\cos \varphi_{j+1}\right)
$$$$
a_{4}=\frac{6 E I\left(\varphi_{j+1}-\sin \varphi_{j+1}\right)}{l^{2}+12 E I / K G A_{\mathrm{p}}}
$$$$
\mathbf{N}=\left[\begin{array}{cccccc}
h_{1} & 0 & 0 & h_{2} & 0 & 0 \\
0 & h_{3} & h_{4} & 0 & h_{5} & h_{6} \\
0 & h_{7} & h_{8} & 0 & h_{9} & h_{10}
\end{array}\right]
$$$$
\mathbf{D}=\left[\begin{array}{ccc}
E A_{\mathrm{p}} & 0 & 0 \\
0 & E I & 0 \\
0 & 0 & K G A_{\mathrm{p}}
\end{array}\right]
$$$$
\mathbf{N}_{u}=\left[\begin{array}{llllll}
h_{1} & 0 & 0 & h_{2} & 0 & 0
\end{array}\right]
$$$$
\mathbf{N}_{w}=\left[\begin{array}{llllll}
0 & h_{7} & h_{8} & 0 & h_{9} & h_{10}
\end{array}\right]
$$$$
\mathbf{m}_{\mathrm{p}}=\left[\begin{array}{ccc}
m_{\mathrm{p}} & 0 & 0 \\
0 & \rho_{\mathrm{p}} I & 0 \\
0 & 0 & m_{\mathrm{p}}
\end{array}\right], \quad \mathbf{m}_{\mathrm{f}}=\left[\begin{array}{ccc}
m_{\mathrm{f}} & 0 & 0 \\
0 & \rho_{\mathrm{f}} I_{\mathrm{f}} & 0 \\
0 & 0 & m_{\mathrm{f}}
\end{array}\right]
$$ 
and

$$
\mathbf{B}=\left[\begin{array}{cccccc}
\frac{\mathrm{d} h_{1}}{\mathrm{~d} x} & 0 & 0 & \frac{\mathrm{d} h_{2}}{\mathrm{~d} x} & 0 & 0 \\
0 & \frac{\mathrm{d} h_{3}}{\mathrm{~d} x} & \frac{\mathrm{d} h_{4}}{\mathrm{~d} x} & 0 & \frac{\mathrm{d} h_{5}}{\mathrm{~d} x} & \frac{\mathrm{d} h_{6}}{\mathrm{~d} x} \\
0 & h_{3}+\frac{\mathrm{d} h_{7}}{\mathrm{~d} x} & h_{4}+\frac{\mathrm{d} h_{8}}{\mathrm{~d} x} & 0 & h_{5}+\frac{\mathrm{d} h_{9}}{\mathrm{~d} x} & h_{6}+\frac{\mathrm{d} h_{10}}{\mathrm{~d} x}
\end{array}\right]
$$

The structural matrices and forcing vector are updated as the nodal coordinates are updated by using a coordinate transformation matrix such as

$$
\mathbf{T}_{i}=\left[\begin{array}{cccccc}
\cos \varphi_{i} & -\sin \varphi_{i} & 0 & 0 & 0 & 0 \\
\sin \varphi_{i} & \cos \varphi_{i} & 0 & 0 & 0 & 0 \\
0 & 0 & 1 & 0 & 0 & 0 \\
0 & 0 & 0 & \cos \varphi_{i+1} & -\sin \varphi_{i+1} & 0 \\
0 & 0 & 0 & \sin \varphi_{i+1} & \cos \varphi_{i+1} & 0 \\
0 & 0 & 0 & 0 & 0 & 1
\end{array}\right]
$$

